\title{
Calidad, satisfacción y valor percibido de los usuarios de un servicio deportivo público
}

\author{
Alberto Nuviala Nuviala* \\ Raquel Pérez-Ordás** \\ Miguel Boceta Osuna ${ }^{* * *}$ \\ Alberto Grao-Cruces ${ }^{* * * *}$ \\ Román Nuviala Nuviala***** \\ José Antonio González Jurado ${ }^{* * * * * *}$
}

\begin{abstract}
Resumen: El objetivo del estudio fue evaluar y establecer modelos de predicción de la calidad percibida, satisfacción y valor percibido de los usuarios de un servicio deportivo. La muestra estuvo formada por 646 usuarios. Cada sujeto cumplimentó un cuestionario cuyas propiedades psicométricas le otorgaron validez. Los resultados obtenidos revelaron que la calidad del servicio deportivo recibido fue buena, resultando los factores técnicos y personal los mejor valorados y siendo la información proporcionada a los usuarios el punto más débil. También positiva fue la valoración de la satisfacción y del valor percibido y dependieron principalmente de un factor intangible como las actividades.

Palabras clave: Deportes. Gestión de calidad. Satisfacción de los consumidores. Políticas públicas.
\end{abstract}

\footnotetext{
*Departamento de Deporte. Universidad Pablo de Olavide. Sevilla, España. E-mail: anuvnvuv@upo.es

**Departamento de Deporte. Universidad Pablo de Olavide. Sevilla, España. E-mail: rperord@upo.es

${ }^{* * \star}$ Departamento de Deporte. Universidad Pablo de Olavide. Sevilla,, España. E-mail: m.boceta@gmail.com

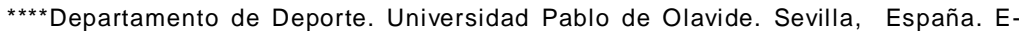
mail:agracru@upo.es

${ }^{* * * * \star D e p a r t a m e n t o ~ d e ~ D e p o r t e . ~ U n i v e r s i d a d ~ P a b l o ~ d e ~ O l a v i d e . ~ S e v i l l a,, ~ E s p a n ̃ a . ~ E-m a i l ~: ~}$ romanescarbo@hotmail.com

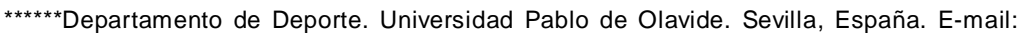
jagonjur@upo.es
} 


\section{INTRODUCCIÓN}

En el mundo empresarial el interés por la gestión de la calidad se ha incrementado en los últimos años, llegando a ser un concepto universalmente conocido y aplicado en todos los ámbitos de la dirección de organizaciones y empresas de servicios (MAÑAS $e t$ al., 2008; MARTÍNEZ; MARTÍNEZ, 2008; TSITSKARI; TSIOTRAS; TSIOTRAS, 2006). No es de extrañar esta situación, ya que medir las percepciones de la calidad del servicio de los usuarios se considera un factor relevante para explicar la competitividad y la viabilidad de las organizaciones, así como conocer el origen o causa de las experiencias positivas de los usuarios (MAÑAS et al., 2008), aspecto fundamental en la implementación de cualquier tipo de sistema que pretenda mejorar la eficiencia y eficacia de los procesos y conseguir la fidelidad de unos usuarios (LARSON; STEINMAN; 2009; MARTÍNEZ; MARTÍNEZ, 2008) cada vez más exigentes (MARTIN; O'NEILL, 2010), se convierte en uno de los mayores éxitos de toda organización (TSITSKARI; TSIOTRAS; TSIOTRAS, 2006).

A pesar de que las organizaciones deportivas tienen que atender a una estructura y funcionamiento diferente al resto de organizaciones (CALISKAN, 2009), no son distintas en cuanto a la necesidad que tienen de evaluar continuamente la calidad (KOO et al., 2009; LARSON; STEINMAN, 2009). La competitividad entre las organizaciones deportivas las conduce al establecimiento de estrategias de diferenciación basadas en la mejora de la satisfacción de los usuarios (MORALES; HERNÁNDEZ-MENDO; BLANCO, 2005), lo que se hace menos difícil cuando se conocen las expectativas de estos y qué consideran importante para la calidad (TSITSKARI et al., 2009). Este es un sector emergente y competitivo (LANGVINIENE; SEKLIUCKIENE, 2008; RIAL et al., 2010) que se ha incorporado con cierto retraso a esta tendencia hacia la calidad (MARTÍNEZ; MARTÍNEZ, 2008), y aunque se haya afirmado que ha alcanzado un nivel similar al de otros sectores y ámbitos económicos (GARCÍA, 2009), la medición de la calidad en la industria 
deportiva aún se encuentra en una etapa formativa (SZABÓ, 2010; TSITSKARI: TSIOTRAS; TSIOTRAS, 2006), precisándose de más investigaciones al respecto (MARTIN; O'NEILL, 2010).

Un análisis de la literatura muestra estudios de calidad en servicios deportivos de rugby (BISSCHOFF; LOTRIET, 2009), deportes acuáticos (CALABUIG; QUINTANILLA; MUNDINA, 2008; DONNE, 2009; LANGVINIENE; SEKLIUCKIENE, 2008), esquí (KYLE et al., 2010; THEODORAKIS; TSIGILIS; KOSTAS, 2009), golf (LEE et al. 2011), atletismo ( UNRUH et al., 2005), spinning (SANZ et al., 2005) o deportes de turismo activo (SHONK; CHELLADURAI, 2008; THWAITES; CHADWICK, 2005). Existen igualmente estudios que analizaron la calidad percibida por espectadores de eventos deportivos (CALABUIG et al, 2010; FUNK; JAM, 2006; LARSON; STEINMAN, 2009; YOSHIDA; JAMES, 2010; YOSHIDA; JAMES, 2011). Pero la mayor parte de los estudios existentes se han centrado en el análisis de la calidad del servicio de centros deportivos (AFTHINOS; THEODORAKIS; NASSIS, 2005; BODET, 2006; GRAMMATIKOPOULOS; PAPACHARISIS; KOUSTELIOS, 2006; SZABÓ, 2010), de naturaleza pública (MORALES et al., 2005; MURRAY; HOWAT, 2002; SANZ; PONCE; RHODEN, 2006; YILDIZ; KARA, 2009) o privada (RIAL et al., 2010; RODRÍGUEZ et al., 2008), sin diferenciar, salvo excepción en escolares (NUVIALA et al., 2011), en función del tipo de práctica físico deportiva de los grupos de usuarios. Esta limitación de los estudios impide comparar los niveles de calidad percibida de los usuarios de los diferentes grupos de actividad, dificultando la toma de decisiones a los responsables de las organizaciones deportivas a fin de mejorar la percepción del servicio prestado.

Las investigaciones indican que la calidad percibida del servicio se determina principalmente por los elementos tangibles de las instalaciones y las actitudes y habilidades de los empleados (AFTHINOS et al., 2005; BODET, 2006; KIM; TRAIL, 2010), aunque recientes estudios relegan a un segundo plano los aspectos 
de interacción social entre empleados y usuarios, atribuyendo una mayor importancia a los diferentes elementos tangibles (MAÑAS et al., 2008; RIAL et al., 2010; SANZ; PONCE; RHODEN, 2006). Independientemente de los elementos que la determinen, lo que sí está bien documentada es la relación de la calidad del servicio como antecedente directo de la satisfacción de los usuarios (BISSCHOFF; LOTRIET, 2009; KYLE et al., 2010; MURRAY; HOWAT, 2002; SHONK; CHELLADURAI, 2009) y esta con las futuras intenciones de los usuarios actuales (KIM; TRAIL, 2010; KYLE et al., 2010; MURRAY; HOWAT, 2002; SHONK; CHELLADURAL, 2008) como potenciales (BISSCHOFF; LOTRIET, 2009). El valor percibido, variable resultante de una evaluación combinada de la percepción de los beneficios y sacrificios (BOKSBERGER; MELSEN, 2011), también ha sido señalado como un mediador importante entre la calidad del servicio y la satisfacción de los usuarios, resultando determinante para las intenciones futuras de los usuarios (CALABUIG et al., 2010; MCDOUGALL; LEVESQUE, 2000; MURRAY; HOWAT, 2002). Ha sido demostrada la influencia positiva y conjunta que estos tres constructos (calidad, satisfacción y valor) tienen sobre la conducta de los usuarios y en la fidelidad de estos hacia el servicio (BRADY et al., 2005).

A pesar de la relación reseñada entre satisfacción y calidad del servicio, se ha cuestionado qué atributos del servicio deben considerarse en la evaluación de la satisfacción y qué dimensiones en la de la calidad (BODET, 2006). El concepto de calidad incluye varias dimensiones (MURRAY; HOWAT, 2002) que responden a una actitud duradera, frente al juicio transitorio afectivo y cognitivo ante un servicio específico que conceptualiza a la satisfacción (VARELA; RIAL; GARCÍA, 2003). Se ha llegado a proponer medir satisfacción conjuntamente con calidad (BISSCHOFF; LOTRIET, 2009), sin diferenciar ambos conceptos (LILJANDER, 1994). Sin embargo, estudios que han medido calidad, satisfacción y valor del servicio sí que han diferenciado los instrumentos con los que valoraron cada una de estas variables (CALABUIG et al., 2010; MCDOUGALL; LEVESQUE, 2000; MURRAY; HOWAT, 2002). 
Siendo el modelo SERVQUAL el instrumento utilizado para la calidad del servicio (BISSCHOFF; LOTRIET, 2009; LARSON; STEINMAN, 2009; LIU, TAYLOR; SHIBLI, 2009; PÉREZ; MINGUET; FREIRE, 2010), aunque no libre de crítica o aportaciones de mejora (ALEXANDRIS et al., 2004; DONNE, 2009; MORALES; HERNÁNDEZ-MENDO; BLANCO, 2009; YOSHIDA; JAMES, 2011) como en diferentes escalas de evaluación para centros deportivos (AFTHINOS et al., 2005; GRAMMATIKOPOULOS et al., 2006; KOO et al., 2009; MORALES et al., 2005; RIAL et al., 2010; YILDIZ; KARA, 2009) o deportes concretos (CALABUIG et al., 2008; LEE et al., 2011; SANZ et al., 2005), así como otros métodos innovadores (MARTÍNEZ; MARTÍNEZ, 2009; MARTÍNEZ; YONG; MARTÍNEZ, 2010; SHONK; CHELLADURAI, 2009) han sido referidos para tal fin, aunque no siempre libre de limitaciones (ALEXANDRIS et al., 2004; CALABUIG et al., 2008).

La variedad de instrumentos de media dificulta la comparación entre diferentes organizaciones, servicios que prestan y actividades realizadas por los usuarios. Al tiempo que no se tienen precedentes de estudios sobre calidad que comparen grupos de actividades deportivas, menos aún que profundicen a su vez en la satisfacción de los usuarios y valor percibido del servicio. Por ello se han marcado como objetivos de esta investigación evaluar la calidad percibida, satisfacción y valor percibido de los usuarios del Instituto Municipal de Deportes de Sevilla (IMD), diferenciando por grupo de actividad practicada y establecer modelos de predicción para la satisfacción y el valor percibido.

\section{MÉTOdo}

\subsection{SuJETOS}

La población objeto de estudio de esta investigación han sido los 13641 usuarios inscritos en actividades dirigidas del IMD de Sevilla que realizan actividades en los centros deportivos gestionados de 
forma directa por el propio servicio, tomando como referencia el mes de abril de 2010. Ante la imposibilidad de encuestar al total de la población se realizó un muestreo proporcional al número de usuarios en cada instalación. Tras la realización del trabajo de campo se obtuvieron un total de 646 cuestionarios, de los cuales el $35.3 \%$ fueron hombres y $64.7 \%$ mujeres, con una edad media de $47.78 \pm 20.41$, lo que permite afirmar que trabajamos con un margen de error $3.84 \%$ para un nivel de confianza del $95 \%$.

De estos sujetos, el $24.2 \%$ tenía estudios universitarios, el $34.8 \%$ educación secundaria, y el $25.6 \%$ estudios básicos, carecía de estudios el porcentaje restante. El 30.6\% de los participantes era pensionista, el $29.8 \%$ trabajador en activo, el porcentaje de parados ascendía al $7.7 \%$, el $14.4 \%$ era estudiante, mientras que había un $16.1 \%$ de sujetos que se dedicaban a las labores del hogar. En términos de frecuencia de actividad, el $13.6 \%$ usaba la organización cuatro o más días por semana, el $82.6 \%$ dos o tres días semanales, y 3.8\% una vez por semana o menos. El tiempo dedicado a cada sesión era de una hora para la mayoría de los sujetos $(75.3 \%)$.

\subsection{INSTRUMENTO}

Para la obtención de datos se utilizó el cuestionario EPOD2 (Nuviala et al., en prensa) compuesto por un total de 25 ítems de respuesta alternativa Likert, que oscilaba entre 1 (muy en desacuerdo) y 5 (muy de acuerdo), con distintas áreas de evaluación:

1. Calidad percibida (20 ítems)

2. Satisfacción (4 ítems)

3. Valor del servicio (1 ítem)

La escala de calidad percibida estaba conformada por seis factores, siendo la fiabilidad del instrumento, medida con alfa de Cronbach, de .924 para el total de la escala (EPOD); .895 para el factor Técnicos; .822 para el factor Personal de Servicios; .716 para el factor Comunicación; .812 para el factor Actividad; .897 para el 
factor Material y .865 para el factor Espacios. La fiabilidad de la escala de satisfacción, conformada por un solo factor, fue de .932.

\subsection{Procedimiento}

El trabajo de campo se realizó mediante un cuestionario autoadministrado con presencia del encuestador. Se solicitó a los participantes que lo cumplimentaran y que consultaran cualquier duda que tuvieran con los ítems. El tiempo invertido en la realización fue de unos 15 minutos. Antes de proceder a la recogida de datos, se pidió permiso a los responsables del IMD de Sevilla. De la misma forma todos los deportistas aceptaron participar voluntariamente en el estudio.

\subsection{Estadística}

Para analizar los datos, tras ser tabulados y mecanizados informáticamente, se utilizó el paquete informático SPSS. Se han pasado las pruebas de normalidad y heterocedasticidad, resultado que los datos obtenidos no se ajustan a la normalidad y que se trata de datos homogéneos, por lo que los análisis estadísticos indicados en este caso son pruebas no paramétricas. Sin embargo, debido a la cantidad de participantes en el estudio se optó por realizar la prueba Anova puesto que es sabido que en grandes poblaciones, aunque se aparten de la normalidad, este estadístico se comporta perfectamente. Utilizándose como variables dependientes la calidad percibida, satisfacción y valor percibido, y como independientes el grupo de actividades practicadas por los usuarios.

Posteriormente se ha realizado un análisis de regresión lineal múltiple, por el método de pasos sucesivos, con el objeto de determinar una ecuación (modelo) que explique el comportamiento de las variables dependientes, satisfacción y valor percibido, utilizando la información proporcionada por variables explicativas o independientes. Se establecieron dos modelos a partir de las diferentes dimensiones de la calidad percibida. 


\section{Resultados}

El fitness es el servicio deportivo más utilizado por los usuarios del IMD de Sevilla en estas instalaciones. En segundo plano encontramos los usuarios de natación y en tercer plano se sitúan los practicantes de deportes de raqueta. En la tabla 1 se muestra la distribución por modalidad deportiva, sexo y edad de los sujetos que han compuesto la muestra de estudio (tabla 1). Los participantes en deportes de raqueta tenían mayor nivel educativo que los que practicaban actividades de fitness o natación. No se encontraron diferencias significativas entre la ocupación laboral de los practicantes de las distintas modalidades deportivas. Ni tampoco en la frecuencia ni en el tiempo de práctica en función de esta división.

Tabla 1- Porcentaje y características de los usuarios por modalidad deportiva practicada.

\begin{tabular}{lcccc}
\hline Modalidad deportiva & Sujetos de la Muestra & Edad & Hombres & Mujeres \\
\hline Fitness & $51.4 \%$ & $50.0 \pm 18.5$ & $25 \%$ & $75 \%$ \\
Deportes de raqueta & $3.3 \%$ & $31.4 \pm 15.0$ & $63.2 \%$ & $36.8 \%$ \\
Natación & $45.3 \%$ & $45.9 \pm 22.0$ & $47.7 \%$ & $52.3 \%$ \\
\hline
\end{tabular}

La valoración media de la calidad percibida obtenida con la

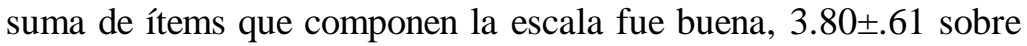
un máximo de 5. Fue la dimensión "técnicos" la que ha obtenido una evaluación más alta por parte de los usuarios, seguido la del personal de servicios, mientras que los factores "comunicación" y "material" han sido los peor calificados. No se han encontrado diferencias 
significativas entre los diferentes grupos de actividades deportivas prestadas por el IMD en los factores "técnicos", "comunicación", "material" y "espacios". Por el contrario, sí las ha habido en los factores "personal de servicios" y "actividad" (tablas 2 y 3 ).

Tabla 2. Calidad percibida (EPOD), Satisfacción y Valor percibido. Diferencias en función de la modalidad deportiva

\begin{tabular}{lllclc}
\hline & & Media & $\begin{array}{c}\text { Desviación } \\
\text { típica }\end{array}$ & F & Sig. \\
& & & & & \\
\hline \multirow{3}{*}{ EPOD } & Fitness & 3.8156 & .62420 & & \\
& Deportes de raqueta & 3.6906 & .53084 & .310 & .734 \\
& Natación & 3.8076 & .61722 & & \\
& Total & 3.8084 & .61799 & & \\
\hline \multirow{3}{*}{ VALOR } & Fitness & 4.0063 & .85645 & & \\
& Deportes de raqueta & 3.7000 & .80131 & \multirow{2}{*}{4.618} & \\
& Natación & 3.7834 & 1.03735 & & \\
& Total & 3.8963 & .94608 & & \\
\hline \multirow{2}{*}{ SATISFACCIÓN } & Fitness & 4.2053 & .72446 & & \\
& Deportes de raqueta & 4.0263 & .61742 & \multirow{2}{*}{1.906} & \\
& Natación & 4.0901 & .81010 & & \\
& Total & 4.1481 & .76234 & & \\
\hline
\end{tabular}

La satisfacción de los usuarios fue muy buena (4.14 .76), no existiendo diferencias significativas entre los diferentes grupos de actividades (tabla 2).

El valor percibido del servicio obtuvo una puntuación media de 3.89 .94 , siendo las actividades de Fitness las que han obtenido una valoración más alta, mientras que los deportes de raqueta la más baja (tabla 2). 
Tabla 3. Valoración de las dimensiones de calidad percibida realizadas por los usuarios. Diferencias en función de la modalidad deportiva practicada.

\begin{tabular}{|c|c|c|c|c|c|}
\hline & & Media & Desviación típica & $\mathbf{F}$ & Sig. \\
\hline TECNICOS & $\begin{array}{l}\text { Fitness } \\
\text { Deportes de raqueta } \\
\text { Natación } \\
\text { Total }\end{array}$ & $\begin{array}{r}4.2674 \\
4.2750 \\
4.1635 \\
4.2221\end{array}$ & $\begin{array}{l}.70661 \\
.66342 \\
.79351 \\
.74529\end{array}$ & 1.450 & .235 \\
\hline $\begin{array}{l}\text { PERSONAL } \\
\text { SERVICIOS }\end{array}$ & $\begin{array}{l}\text { Fitness } \\
\text { Deportes de raqueta } \\
\text { Natación } \\
\text { Total }\end{array}$ & $\begin{array}{l}4.0094 \\
3.4737 \\
4.0727 \\
4.0218\end{array}$ & $\begin{array}{l}.84337 \\
.63407 \\
.79759 \\
.82238\end{array}$ & 4.856 & .008 \\
\hline COMUNICACION & $\begin{array}{l}\text { Fitness } \\
\text { Deportes de raqueta } \\
\text { Natación } \\
\text { Total }\end{array}$ & $\begin{array}{l}3.4327 \\
3.4833 \\
3.5377 \\
3.4818\end{array}$ & $\begin{array}{l}.88803 \\
.93330 \\
.78628 \\
.84520\end{array}$ & 1.127 & .325 \\
\hline ACTIVIDAD & $\begin{array}{l}\text { Fitness } \\
\text { Deportes de raqueta } \\
\text { Nat ación } \\
\text { Total }\end{array}$ & $\begin{array}{l}3.9994 \\
3.7158 \\
3.8742 \\
3.9352 \\
\end{array}$ & $\begin{array}{l}.66961 \\
.62295 \\
.67993 \\
.67567\end{array}$ & 3.531 & .030 \\
\hline MATERIAL & $\begin{array}{l}\text { Fitness } \\
\text { Deportes de raqueta } \\
\text { Natación } \\
\text { Total }\end{array}$ & $\begin{array}{l}3.5338 \\
3.3684 \\
3.6131 \\
3.5646\end{array}$ & $\begin{array}{l}1.04153 \\
.98692 \\
.91808 \\
.98546\end{array}$ & .861 & .423 \\
\hline ESPACIOS & $\begin{array}{l}\text { Fitness } \\
\text { Deportes de raqueta } \\
\text { Natación } \\
\text { Total }\end{array}$ & $\begin{array}{l}3.6591 \\
3.3158 \\
3.7395 \\
3.6853\end{array}$ & $\begin{array}{l}.85539 \\
.67104 \\
.86074 \\
.85505\end{array}$ & 2.493 & .084 \\
\hline
\end{tabular}

Para comprender el valor percibido de los usuarios se ha realizado un análisis de regresión (tabla 4) mediante el método de pasos sucesivos que da como resultado la existencia de dos modelos. El modelo final ha seleccionado dos variables, actividad y comunicación, con un $\mathrm{F}=138,628$; $\mathrm{p}<.001$, lo que nos permite afirmar que existe una relación lineal significativa entre la variable dependiente y las variables introducidas. La varianza explicada es de $33.40 \%$. 
Puede observarse que la variable más importante en el modelo de Valor percibido fue actividad, con un valor Beta de .473, mientras que comunicación presentó un valor Beta de .151.

Tabla 4 - Modelo de regresión múltiple predictor del Valor percibido a partir de las dimensiones de la calidad percibida

\begin{tabular}{|c|c|c|c|c|c|c|c|c|c|}
\hline \multirow[b]{2}{*}{ Modelo } & \multirow[b]{2}{*}{$\mathrm{R}$} & \multirow[b]{2}{*}{ R cuadrado } & \multirow{2}{*}{\multicolumn{2}{|c|}{ 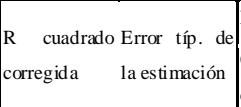 }} & \multicolumn{5}{|c|}{ Estadísticos de cambio } \\
\hline & & & & & $\begin{array}{l}\text { Cambio en } \mathrm{R} \\
\text { cuadrado }\end{array}$ & Cambio en F & gl1 & g12 & $\begin{array}{l}\text { Sig. Cambio } \\
\text { en F }\end{array}$ \\
\hline 2 & .578 & .334 & 332 & .76326 & 014 & 11,758 & 1 & 553 & 001 \\
\hline
\end{tabular}

Variables predictoras: (Con stante), ACTIVIDAD, COMUNICACIÓN

Coeficientes

\begin{tabular}{|c|c|c|c|c|c|c|}
\hline & & \multicolumn{2}{|c|}{ Coeficientes no estandarizados } & \multirow{2}{*}{\begin{tabular}{|l|}
$\begin{array}{l}\text { Coeficientes } \\
\text { tipificados }\end{array}$ \\
Beta
\end{tabular}} & \multirow[b]{2}{*}{$\mathrm{t}$} & \multirow[b]{2}{*}{ Sig. } \\
\hline \multicolumn{2}{|c|}{ Modelo } & B & Error típ. & & & \\
\hline \multirow[t]{3}{*}{2} & (Constante) & 704 &, 194 & & 3,621 &, 000 \\
\hline & ACTIVIDAD &, 662 &, 061 & .473 & 10,774 &, 000 \\
\hline & COMUNICACIÓN &, 165 &, 048 &, 151 & 3,429 &, 001 \\
\hline
\end{tabular}

Variable dependiente: VALOR

El modelo de Satisfacción se ha realizado por medio de un análisis de regresión por pasos sucesivos (tabla 5) que da como resultado la existencia de seis modelos. El modelo final ha seleccionado todas las variables, excepto comunicación, presentando un $\mathrm{F}=114,568 ; \mathrm{p}<.001$, lo que nos permite afirmar que existe una relación lineal significativa entre la variable dependiente y las variables introducidas. La varianza explicada es de $55.6 \%$.

Puede observarse que las variables más importantes en el modelo de Satisfacción son actividad, con un valor Beta de .347, y técnicos, con un valor Beta de.266. Valor y material son las variables menos importantes, ambas con un valor Beta de .074 (tabla 5). 
Tabla 5 - Modelo de regresión múltiple predictor de Satisfacción del servicio a partir de las dimensiones de la calidad percibida y Valor percibido

Resumen del modelo

\begin{tabular}{|c|c|c|c|c|c|c|c|c|c|c|}
\hline Modelo & $\mathrm{R}$ & $R$ cuadrado & $\begin{array}{l}\mathrm{R} \quad \text { cuadrado } \\
\text { corregida }\end{array}$ & $\begin{array}{l}\text { Error típ. de } \\
\text { la estimación }\end{array}$ & \begin{tabular}{|l|} 
Estadísticos de \\
Cambio en R \\
cuadrado
\end{tabular} & Cambio en F & g11 & g12 & $\begin{array}{l}\text { Sig. } \\
\text { en F }\end{array}$ & Cambio \\
\hline$\overline{6}$ &, 746 & ,556 &, 552 & 50795 &, 003 & 4,075 & 1 & 548 &, 044 & \\
\hline
\end{tabular}

Variables predictoras: (Constante), ACTIVIDAD, TECNICOS, ESPACIOS, PPAS, VALOR, MATERIAL

Coeficientes

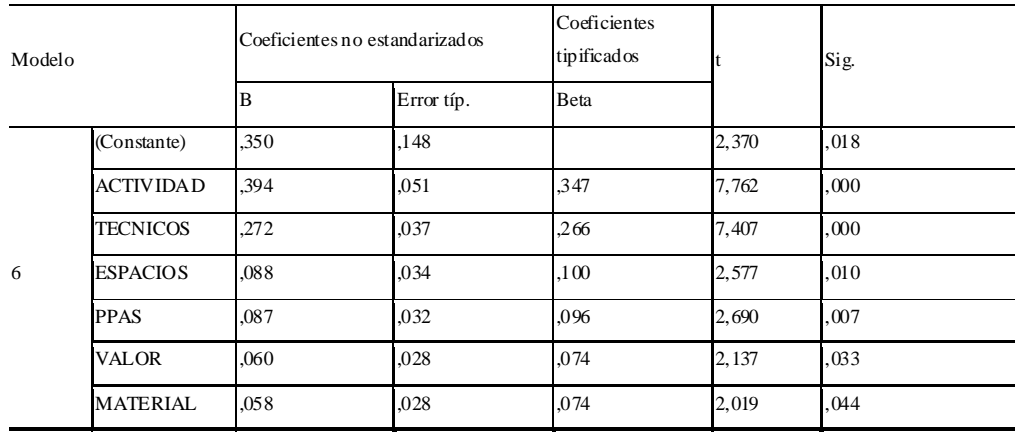

Variable dependiente: SATISFACCIÓN

\section{DisCUSIÓN Y CONCLUSIONES}

La demanda principal de servicios al IMD de Sevilla se dirige hacia las actividades relacionadas con el fitness y la natación. Los deportes de raqueta se encuentran muy lejos en cuanto a demanda. Resultados que están en consonancia con los estudios sobre hábitos deportivos en España (CENTRO DE INVESTIGACIONES SOCIOLÓGICAS, 2010), que encontraron las mayores tasas de práctica para las actividades de fitness (29.9\%) y al auge de la participación en actividades de fitness en Europa, que se acompaña de un estancamiento de la práctica de deportes tradicionales (FRIDBERG, 2010). Es necesario mencionar la importancia de este dato, puesto que los jóvenes europeos son más propensos a ser miembros de centros de fitness que los de mayor edad (COMISIÓN EUROPEA, 2010). Tendencia que no se contradice con los resultados obtenidos, donde entre los sujetos de mayor edad también prevalece este tipo de actividades. 
En relación a la valoración de la calidad del servicio, se puede afirmar que ha sido positiva, al obtener una puntuación media para el total de los ítems de 3.81土.61 sobre un máximo de 5.00 puntos. Resultó que las dimensiones vinculadas con los recursos humanos fueron las mejores valoradas, "técnicos" (4.22士.74) y "personal de servicios" (4.02 \pm .82$)$, mientras que la comunicación fue el factor que obtuvo una peor valoración (3.48土.84). Resultados acordes con los hallados por Afthinos et al. (2005), Mañas et al. (2008), Nuviala et al. (2011) y Rial et al. (2010).

No se han encontrado diferencias significativas en la valoración de la calidad del servicio recibido en función de las actividades realizadas por los usuarios, contrariamente a lo que se había hallado en población escolar (NUVIALA et al., 2011). No se tienen precedentes de estudios que comparen en población adulta la calidad percibida por usuarios en función de los grupos de actividad realizada por estos, lo que unido a la variedad de instrumentos de medida para la calidad impide la comparación entre los estudios existentes. Por lo que partiendo de la importancia por conocer al usuario (BEDNARIK; SUGMAN; URANK; KOVAC, 2007) con el objeto de tomar medidas para mejorar los servicios deportivos (BEDNARIK; KOLAR; JURAK, 2010), estos resultados permiten establecer diferentes estrategias de mejora de la valoración de las diferentes actividades, lo que base a la literatura existente tenderían a derivar en una mayor adherencia a los servicios deportivos por parte de los usuarios actuales o futuros (BISSCHOFF; LOTRIET, 2009; KIM; TRAIL, 2010; KYLE et al., 2010; MURRAY; HOWAT, 2002; SHONK; CHELLADURAI, 2008).

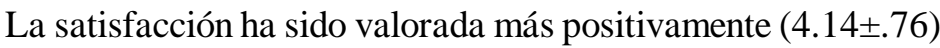
que su antecedente, la calidad del servicio (BISSCHOFF; LOTRIET, 2009; KYLE et al., 2010; MURRAY; HOWAT, 2002; SHONK; CHELLADURAI, 2009). No se han encontrado diferencias significativas entre los diferentes grupos de actividades para esta variable. Se puede observar una relación entre la calidad percibida y la satisfacción puesto que la actividad mejor valorada cuenta con los usuarios más satisfechos, y la actividad peor valorada cuenta con 
los usuarios menos satisfechos. Sin embargo los resultados obtenidos en la valoración de la calidad y la satisfacción muestran diferencias en términos absolutos siendo mayor la satisfacción que la calidad. Estos resultados podrían explicarse por las peculiaridades de la calidad, actitud duradera a lo largo del tiempo, y de la satisfacción, juicio transitorio ante un servicio específico (VARELA; RIAL; GARCÍA, 2003), lo que hace pensar que el juicio de la satisfacción depende en gran medida de aspectos emocionales (OLSEN; JOHNSON, 2003).

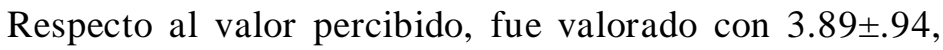
encontrándose diferencias significativas $(\mathrm{p}<.001)$ en función del grupo de actividad físico deportiva de los usuarios. Al igual que ha ocurrido con calidad percibida y satisfacción, a pesar de no existir diferencias significativas entre actividades, ha sido el fitness la actividad que mejor valor percibido ha tenido, mientras que deportes de raqueta es el que peor resultado ha obtenido Estos resultados refuerzan la relación positiva existente entre la satisfacción de los usuarios y el valor percibido (CALABUIG et al., 2010; MCDOUGALL; LEVESQUE, 2000; MURRAY; HOWAT, 2002).

Estudiada la valoración de la calidad, valor percibido y satisfacción por los usuarios de los diferentes grupos de actividad, es necesario comprender el modelo predictivo de valor percibido. El análisis de regresión para esta variable mostró a la dimensión actividades como la que más porcentaje explica del valor percibido $(\mathrm{R} 2=.320)$, al igual que ocurrió para el modelo predictor de la satisfacción. Solamente se incluyó posteriormente la dimensión comunicación en el modelo predictivo realizado mediante el procedimiento de pasos sucesivos, incrementando así el porcentaje de la varianza en un 1.4\%, llegando a explicar el $33.4 \%$ de la varianza final, inferior al explicado mediante ecuaciones estructurales por Murray y Howatt (2002). En la ecuación de regresión (valor percibido $=.704+.662$ (actividades) +.165 (comunicación)) la variable actividades, con un valor Beta de .472 , fue el mejor predictor del valor percibido, mientras que comunicación presentó un valor Beta de .151. Estos resultados no coinciden con los reportados por la literatura, la cual señala al factor humano como el principal predictor 
de la calidad percibida y por tanto del valor percibido, consecuencia de la calidad del servicio (BODET, 2006; MURRAY; HOWAT, 2002), así como a los elementos tangibles cuya importancia no ha hecho más que incrementar en los últimos años (MAÑAS et al., 2008; RIAL et al., 2010).

Por lo que se refiere al modelo predictivo de satisfacción en el que hemos incluido el valor percibido como elemento que condiciona la satisfacción (MURRAY; HOWATT, 2002), mostró que la dimensión actividades es la que explica el mayor porcentaje ( $\mathrm{R} 2=.466)$, mientras que el resto de variables incluidas, todas excepto comunicación, suponen un incremento de un 9\%. El modelo final $($ satisfacción $=.350+.394$ (actividades $)+.272($ técnicos $)+.088$ $($ espacios $)+.087$ (personal de servicios $)+.060$ (valor) +.058 (material)) que explica un $55.6 \%$ de la varianza. Las dimensiones actividades y técnicos son las más relevantes en la ecuación de regresión, al poseer unos valores Beta de .347 y .266 respectivamente, relegando a las variables valor y material como la menos importantes, con un valor Beta de .074.

La aplicación práctica del estudio es el establecimiento de estrategias de gestión para la mejora de la calidad del servicio, valor percibido y satisfacción de los usuarios de organizaciones deportivas, cuyo culmen se encuentra en conseguir la fidelidad estos usuarios (BRADY et al., 2005; CALABUIG et al., 2010; KIM; TRAIL, 2010; KYLE et al., 2010; MCDOUGALL; LEVESQUE, 2000; MURRAY; HOWAT, 2002; SHONK; CHELLADURAI, 2008) y atraer a usuarios potenciales (BISSCHOFF; LOTRIET, 2009).

Se concluye que la calidad del servicio deportivo recibido fue buena, resultando los factores técnicos y personal de servicios los mejor valorados y siendo la información proporcionada a los usuarios el punto más débil. También positiva fue la valoración de la satisfacción y del valor percibido por el usuario. Solamente se encontraron diferencias significativas en estos tres constructos en función del grupo de actividad en el valor percibido. Las actividades de fitness fueron las mejor puntuadas y las que recibieron la puntuación menos positiva fueron los deportes de raqueta. Como 
muestran los modelos de regresión, tanto la satisfacción con el servicio como el valor percibido del mismo dependieron principalmente de un factor intangible como las actividades. Futuros estudios deberían atender a las variables estudiadas en este trabajo en organizaciones deportivas que presten servicios en contextos diferentes. Dado que los resultados obtenidos pueden ser susceptibles de variación según las características de los sujetos estudiados. 
Quality, satisfaction and perceived value of users of public service sports

Abstract: The objetive of this study was to evaluate the perceived quality, satisfaction and perceived value of the sports service users. The sample consisted of 646 users. Each user filled in a self-administered questionnaire, which gave their valid psychometric properties to assess the perceived quality, satisfaction and perceived value.The results obtained showed that the quality of service received was good sport, being the technical and service personnel the best values and the information being provided to the weakest users. Also positive was the assessment of satisfaction and perceived value to the user. Both satisfaction and perceived value of the service depends primarily on an intangible factor and activities. Keywords: Sports. Quality management. Consumer satifaction. Public policies.

Qualidade, satisfação, e valor percebido de
usuários de esportes serviço público
Resumo: O objetivo deste estudo foi avaliar e
desenvolver modelos para prever a qualidade,
satisfação e valor percebido dos usuários do serviço
desportivo. A amostra consistiu de 646 usuários. Cada
sujeito preencheu um questionário cujas propriedades
psicométricas deu validade. Os resultados obtidos
mostraram que a qualidade do serviço desportivo
recebido foi boa, tendo os factores técnicos os
melhores valores, e as informações que são
fornecidas para os usuários o ponto mais fraco.
Também positiva foi a avaliação de satisfação e do
valor percebido, que dependiam principalmente de um
fator intangível, como são as atividades.
Palabras clave: Esportes. Gestão de qualidade.
Satisfação dos consumidores. Políticas públicas.

\section{REFERENCIAS}

AFTHINOS, Y.; THEODORAKIS N. D.; NASSIS, P. Customers' expectations of service in Greek fitness centers. Managing Service Quality, Bradford, v. 15, n. 3, p. 245258, marzo de 2005.

ALEXANDRIS, K. et al. An empirical investigation into the role of the outcome dimension in measuring perceived service quality in a health club context. International Journal of Sport Management, Champaign, v. 5, n. 3, p. 281294, 2004. 
BEDNARIK, J.; KOLAR, E.; JURAK, G. Analysis of the sports services market in Slovenia. International Journal of Fundamental and Applied Kinesilogy, Zagreb, v. 42, n.2, p. 142-152, 2010.

BEDNARIK, J.; SUGMAN, R.; URANK, J.; KOVAC, M. Segmentation of sports consumers in Slovenia. International Journal of Fundamental and Applied Kinesilogy, Zagreb, v. 391, p. 74-84, 2007.

BISSCHOFF, C. A.; LOTRIET, R. A. The service quality of the PUK rugby institute. Tydskrif Vir Geesteswetenskappe, Arcadia, v. 49, n.2, p. 266-286, 2009.

BODET, G. Investigating customer satisfaction in a health club context by an application of the tetraclasse model. European Sport Management Quarterly, Aachen, v. 6, n.2, p. 149-165, 2006.

BOKSBERGER, P. E.; MELSEN, L. Perceived value: a critical examination of definitions, concepts and measures for the service industry. Journal of Services Marketing, Wichita, v. 25, n. 3, p. 229-240, 2011.

BRADY, M.K. et al. Removing the contextual lens: a multinational, multi-setting comparison of service evaluation models. Journal of Retailing, New York, v. 81, n. 3, p. 215-230, julio de 2005.

CALABUIG, F. et al. Satisfacción, calidad y valor percibido en espectadores de atletismo. Revista Internacional De Medicina y Ciencias De La Actividad Física y El Deporte, Madrid, v. 10, n. 40, p. 577-593, 2010.

CALABUIG, F.; QUINTANILLA, I.; MUNDINA, J. La calidad percibida de los servicios deportivos: diferencias según instalación, género, edad y tipo de usuario en servicios náuticos. International Journal of Sport Science, Madrid, v. 4, n. 10, p. 25-43, enero de 2008 .

CALISKAN, G. Criteria in sport management. International Business Management, Perth, 3, n.2, p. 22-31, 2009.

CENTRO DE INVESTIGACIONES SOCIOLÓGICAS. Encuesta sobre los hábitos deportivos en España 2010. Avance de resultados. En http://www.csd.gob.es/ csd/estaticos/noticias/DOSSIER-ENCUESTA.pdf. Recuperado el 27 de junio de 2011, de 2010.

DONNE, K. ADVENTUREQUAL: An extension of the SERVQUAL conceptual gap model in young people's outdoor adventure. International Journal of Sport Management and Marketing, Lisboa, v. 6, n. 3, p. 253-276, 2009.

EUROPEAN COMMISSION. SPECIAL EUROBAROMETER 334, Sport and Physical Activity. 2010 En http://ec.europa.eu/public_opinion/archives/ebs/ebs_334_en.pdf. Recuperado el 27 de junio de 2011, de 2010.

FRIDBERG, T. Sport and exercise in Denmark, Scandinavia and Europe. Sport in Society, Abingdon, 13, n.4, p. 583-592, 2010. 
FUNK, D. C.; JAM, J. D. Consumer loyalty: The meaning of attachment in the development of sport team allegiance. Journal of Sport Management, Champaign, v. 20, n.2, p. 189-21, 2006.

GARCÍA, J.A. Estudio de la invarianza de escala mediante el método de cálculo integral en la medición de la calidad percibida de los servicios deportivos. RICYDE. Revista Internacional De Ciencias Del Deporte, Madrid, v. 15, p. 17-35, 2009.

GRAMMATIKOPOULOS, V.; PAPACHARISIS, V.;KOUSTELIOS, A. Modeling sport services in greece: A re-evaluation of outcome dimension. Perceptual and Motor Skills, Montana, v. 102, n. 1, p. 62-64, 2006.

KIM, M.; TRAIL, G. T. The effects of service provider employment status and service quality exchange on perceived organizational image and purchase intention. Sport Management Review, Oxford, v. 13, n. 3, p. 225-234, 2010.

$\mathrm{KOO}, \mathrm{G}$ et al. Examination of the causal effects between the dimensions of service quality and spectator satisfaction in minor league baseball. International Journal of Sports Marketing and Sponsorship, London, v. 11, n. 1, p. 46-59, 2009.

KYLE, G. T. et al. The effect of service quality on customer loyalty within the context of ski resorts. Journal of Park \& Recreation Administration, Champaign, v. 28 , n. 1, p. 1-15, 2010.

LANGVINIENE, N.; SEKLIUCKIENE, J. Assessment of SPA services quality in lithuania: Customer's viewpoint. Amfiteatru Economic, Bucure?ti, v. 10, n. 2, p. 230-241, 2008.

LARSON, B. V.; STEINMAN, R. B. Driving NFL fan satisfaction and return intentions with concession service quality. Services Marketing Quarterly, Binghamton, v. 30, n. 4, p. 418-428, 2009.

LEE, J. et al. The influence of service quality on satisfaction and intention: A gender segmentation strategy. Sport Management Review, Oxford, v. 14, n. 1, p. 5463, 2011.

LILJANDER, V. Modeling perceived service quality using different comparison standards. Journal of Consumer Satisfaction, Dissatisfaction and Complaining Behaviour, Provo, v. 7, p. 126-142, 1994.

LIU, Y.; TAYLOR, P.; SHIBLI, S. Measuring customer service quality of english public sport facilities. International Journal of Sport Management and Marketing, Lisbon, v. 6, n. 3, p. 229-252, 2009.

MAÑAS, M. A., et al. Tangibles as predictors of customer satisfaction in sports services. Psicothema, Oviedo, v. 20, n. 2, p. 243-248, 2008.

MARTIN, D. S.; O'NEILL, M. Scale development and testing: A new measure of cognitive satisfaction in sports tourism. Event Management, Brisbane, v. 14, n. 1, 1-15, 2010. 
MARTÍNEZ, J. A.; MARTÍNEZ, L. Measuring perceived service quality in sports services; A first person approach. Revista Internacional De Medicina y Ciencias De La Actividad Fisica y Del Deporte, Madrid, v 8, n. 31, p. 244-255, 2008.

MARTÍNEZ, J. A.; MARTÍNEZ, L. Applying brand concept maps to study perceived quality in sports services. Revista Internacional De Medicina y Ciencias De La Actividad Física y Del Deporte, Madrid, v 9, n. 35, p. 232-253, 2009.

MARTÍNEZ, J. A.; YONG, J. K.; MARTÍNEZ, L. An application of fuzzy logic to service quality research: A case of fitness service. Journal of Sport Management, Champaign, v. 24, n. 5, p. 502-523, 2010.

MCDOUGALL, G. H.; LEVESQUE, T. Customer satisfaction with wervice: Putting perceived value into the equation. Journal of Services Marketing, Santa Bárbara, v. 14 , n. 5 , p. $392-410,2000$.

MORALES, V.; HERNÁNDEZ-MENDO, A.; BLANCO, Á. Evaluating qualityinsports organisations: Anadaptation of theservqual model. Revista De Psicologia Del Deporte, Palma de Mallorca, v. 18, n. 2, p. 137-150, 2009.

MORALES, V.; HERNÁNDEZ-MENDO, A.; BLANCO, Á. Quality evaluation in physical activity programs. Psicothema, Oviedo, v. 17, n. 2, p. 311-317, 2005.

MURRAY, D.; HOWAT, G. The relationships among service quality, value, satisfaction, and future intentions of customers at an Australian sports and leisure centre. Sport Management Review, Oxford, v. 5, n. 1, p. 25-43, mayo de 2002.

NUVIALA, A. et al.. Diseño y análisis del cuestionario de valoración de servicios deportivos (EPOD2). Revista Internacional De Medicina y Ciencias De la Actividad Física y El Deporte, Madrid. (en prensa)

NUVIALA, A., et al. Calidad del servicio deportivo en edad escolar desde una doble perspectiva. Revista Internacional de Medicina y Ciencias de la Actividad Física y el Deporte, Madrid, v. 11, n. 42, p. 220-235, junio de 2011.

OLIVER, R. A cognitive model of the antecedents and consequences of satisfaction decisions. Journal of Marketing Research, Chicago, v. 17, p. 460-469, 1980.

OLSEN, L. L.; JOHNSON, M.D. Service equity, satisfaction, and loyalty: from transaction-specific to cumulative evaluation. Journal of Service Research, Thousand Oaks, v. 5, n. 3, p. 184-195, 2003.

PÉREZ, V. R.; MINGUET, J.L.C.; FREIRE, M.G. Sports management services: The dimensions of quality. Journal of Human Sport \& Exercise, Alicante, v. 5, n. 2, p. 295-306, 2010.

RIAL, J. et al. Modelización y medida de la calidad percibida en centros deportivos: La escala QSport-10. RICYDE. Revista Internacional De Ciencias Del Deporte, Madrid, v. 6, n. 18, p. 57-73, 2010. 
SANZ, E.; PONCE, L. E.; RHODEN, I. Satisfaction according to the physical sport activities service of free time. the specific case of the community of the university of la rioja (Spain). Movimento, Porto Alegre, v., 12, n. 3, p. 13-42, 2006.

SANZ, I. et al. La satisfacción en los practicantes de spinning: Elaboración de una escala para su medición; la satisfacción en los practicantes de spinning: Elaboración de una escala para su medición. Motricidad. European Journal of Human Movement, Granada, v. 13, p. 17-36, 2005.

SHONK, D. J.; CHELLADURAI, P. Model of service quality in event sport tourism: Development of a scale. International Journal of Sport Management and Marketing, Lisbon, v. 6, n. 3, p. 292-307, 2009.

SHONK, D. J.; CHELLADURAI, P. Service quality, satisfaction, and intent to return in event sport tourism. Journal of Sport Management, Champaign , v. 22(5), p. 587-602, 2008.

SZABÓ, Á. Leisure sport services quality. INTERNATIONAL CONFERENCE ON SERVICE SYSTEMS AND SERVICE MANAGEMENT, ICSSSM'10, 7. Tokyo: ICSSSM, 2010. p. $821-826$.

THEODORAKIS, N. D.; TSIGILIS, N.; KOSTAS, A. The mediating role of place attachment on the relationship between service quality and loyalty in the context of skiing. International Journal of Sport Management and Marketing, Lisboa, v. 6 , n. 3, p. 277-291, 2009.

THWAITES, D.; CHADWICK, S. Service quality perspectives in sport tourism. Sport in Society, Abingdon, v. 8, n. 2, p. 321-337, 2005.

TSITSKARI, E. et al. Expected and perceived service quality at basketball stadiums in greece. World Leisure Journal, New Zealand v. 51, n. 2, p. 94-104, 2009.

TSITSKARI, E.; TSIOTRAS, D.; TSIOTRAS, G. Measuring service quality in sport services, Total Quality Management \& Business Excellence, Abingdon v. 17, n. 5 , p. $625-631,2006$.

UNRUH, S. et al. Collegiate student-athletes' satisfaction with athletic trainers. Journal of Athletic Training, Dallas, v. 40, n. 1, p. 52-55, 2005.

VARELA, J.; RIAL., A.; GARCÍA, E. Presentación de una escala de satisfacción con los servicios sanitarios de atención primaria. Psicothema, Oviedo, v. 15, n. 4, p. 656-661, 2003.

YILDIZ, S. M.; KARA, A. The PESPERF scale: An instrument for measuring service quality in the school of physical education and sports sciences (PESS). Quality Assurance in Education, Bradford, v. 17, n. 4, p. 393-415. 2009.

YOSHIDA, M.; JAMES, J. D. Customer satisfaction with game and service experiences: Antecedents and consequences. Journal of Sport Management, Champaign, v. 24, n. 3, p. 338-361, 2010. 
YOSHIDA, M.; JAMES, J. D.. Service quality at sporting events: Is aesthetic quality a missing dimension? Sport Management Review, Oxford, v. 14, n. 1, p. 13-24, 2011.

Apoyo: Universidad Pablo de Olavide

Endereço para correspondencia:

Alberto Nuviala Nuviala

Edificio "Conde de Aranda" (Edificio 11)

Planta 2 $2^{\mathrm{a}}$, despacho 5.

Carretera de Utrera Km. 1

41013 - Sevilla, España

Recebido em: 20/10/2011

Aprovado em: 04/04/2012

Movimento, Porto Alegre, v. 18, n. 04, p. 11-32, out/dez de 2012. 
